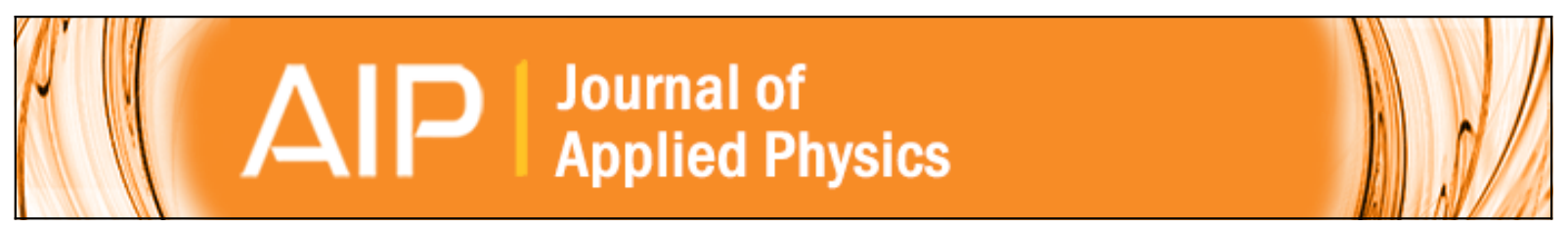

\title{
Conditions for spin-gapless semiconducting behavior in Mn2CoAl inverse Heusler compound
}

\author{
I. Galanakis, K. Özdoan, E. aolu, and S. Blügel
}

Citation: Journal of Applied Physics 115, 093908 (2014); doi: 10.1063/1.4867917

View online: http://dx.doi.org/10.1063/1.4867917

View Table of Contents: http://scitation.aip.org/content/aip/journal/jap/115/9?ver=pdfcov

Published by the AIP Publishing

\section{Articles you may be interested in}

Magneto-transport properties of oriented Mn2CoAl films sputtered on thermally oxidized Si substrates

Appl. Phys. Lett. 104, 242408 (2014); 10.1063/1.4884203

Magnetic and transport properties of Mn2CoAl oriented films

Appl. Phys. Lett. 103, 142403 (2013); 10.1063/1.4823601

Slater-Pauling behavior in LiMgPdSn-type multifunctional quaternary Heusler materials: Half-metallicity, spingapless and magnetic semiconductors

J. Appl. Phys. 113, 193903 (2013); 10.1063/1.4805063

Ab-initio calculations of the electronic and magnetic structures of Co2 $\mathrm{Cr} 1 \times \mathrm{Mn} \times \mathrm{Al}$ alloys

J. Appl. Phys. 112, 123904 (2012); 10.1063/1.4768906

Exploring Co2MnAl Heusler compound for anomalous Hall effect sensors

Appl. Phys. Lett. 99, 132509 (2011); 10.1063/1.3644157

\section{AlP | Journal of}

Journal of Applied Physics is pleased to announce André Anders as its new Editor-in-Chief 


\title{
Conditions for spin-gapless semiconducting behavior in $\mathrm{Mn}_{2} \mathrm{CoAl}$ inverse Heusler compound
}

\author{
I. Galanakis, ${ }^{1, a)}$ K. Özdoğan, ${ }^{2, b)}$ E. Şaşıŏlu, ${ }^{3, c)}$ and S. Blügel ${ }^{3}$ \\ ${ }^{1}$ Department of Materials Science, School of Natural Sciences, University of Patras, GR-26504 Patra, Greece \\ ${ }^{2}$ Department of Physics, Yildiz Technical University, 34210 Istanbul, Turkey \\ ${ }^{3}$ Peter Grünberg Institut and Institute for Advanced Simulation, Forschungszentrum Jülich and JARA, \\ 52425 Jülich, Germany
}

(Received 13 February 2014; accepted 25 February 2014; published online 7 March 2014)

\begin{abstract}
Employing ab initio electronic structure calculations, we investigate the conditions for spin-gapless semiconducting (SGS) behavior in the inverse $\mathrm{Mn}_{2} \mathrm{CoAl}$ Heusler compound. We show that tetragonalization of the lattice, which can occur during films growth, keeps the SGS character of the perfect cubic compound. On the contrary, atomic swaps even between sites with different local symmetry destroy the SGS character giving rise to a half-metallic state. Furthermore, the occurrence of Co-surplus leads also to half-metallicity. Thus, we propose that in order to achieve SGS behavior during the growth of $\mathrm{Mn}_{2} \mathrm{CoAl}$ (and similar SGS Heusler compounds) thin films, one should minimize the occurrence of defects, while small deformations of the lattice, due to the lattice mismatch with the substrate, do not play a crucial role. (C) 2014 AIP Publishing LLC. [http://dx.doi.org/10.1063/1.4867917]
\end{abstract}

\section{INTRODUCTION}

Heusler compounds are a huge family of ternary and quaternary compounds with more than 1000 known members. ${ }^{1}$ Most of them crystallize in cubic structures and often they are magnetic adopting various magnetic orders. ${ }^{1}$ Heusler compounds and alloys have attracted considerable attention during the last two decades due to the discovery of the halfmetallicity ${ }^{2,3}$ in several compounds, which should maximize the performance of spintronic/magnetoelectronic devices. ${ }^{4}$ Half-metallic (HM) magnets present metallic behavior for one spin-direction, while they possess an energy gap around the Fermi level in the other spin direction allowing the creation of $100 \%$ spin-polarized current in devices. ${ }^{2,3}$ Moreover, half-metallicity is always accompanied by the so-called Slater-Pauling rule, where the total spin magnetic moment scales linearly with the number of valence electrons in the unit cell. Variants of such rules have been found for different families of Heusler compounds, i.e., semi-Heuslers like $\mathrm{NiMnSb},{ }^{5}$ full-Heuslers like $\mathrm{Co}_{2} \mathrm{MnAl},{ }^{6}$ inverse full-Heuslers like $\mathrm{Cr}_{2} \mathrm{CoGa}{ }^{7}$ and the quaternary full-Heuslers like $\mathrm{CoFeMnAl}^{8}$

Although HM Heusler compounds have been widely studied, still first-principles calculations predict novel properties among them. For instance, ferromagnetic or ferrimagnetic semiconducting Heusler compounds could be used as spin-filter materials. ${ }^{9,10}$ Another case of interest is the spingapless semiconductors (SGS); magnetic semiconductors where there is an almost vanishing zero-width energy gap at the Fermi level in one spin direction and a usual energy gap in the other spin-direction. ${ }^{11}$ These materials are of special interest since the mobility of carriers is considerably larger

\footnotetext{
a)galanakis@upatras.gr

b)kozdogan@yildiz.edu.tr

c)e.sasioglu@fz-juelich.de
}

than in usual semiconductors and excited carriers (both electrons and holes) can be $100 \%$ spin-polarized simultaneously, leading to new functionalities in devices. The first gapless semiconductors were not magnetic, ${ }^{11-14}$ and it was in 2008 that Wang proposed that the doping of $\mathrm{PbPdO}_{2}$ would lead to a spin gapless semiconductor. ${ }^{15}$ This hypothesis was recently confirmed by Kim and collaborators who studied polycrystalline films of $\mathrm{Mn}$ and $\mathrm{Co}$ doped $\mathrm{PbPdO}_{2} \cdot{ }^{16}$ Other SGS materials include (i) the graphene ribbons altered by $\mathrm{CH}_{2}$ radical groups, ${ }^{17}$ where the magnetism stems from the unsaturated carbon states, (ii) graphene nanoribbons with sawtooth edges under a transverse electrical field, ${ }^{18}$ (iii) the ferromagnetic semiconductor $\mathrm{HgCr}_{2} \mathrm{Se}_{4}$, which becomes SGS under a pressure of $9 \mathrm{GPa},{ }^{19}$ (iv) the $\mathrm{BN}$ nanoribbons with vacancies, ${ }^{20}$ and (v) $\mathrm{Fe}$ and $\mathrm{Cr}$ doped boron nitride sheets. $^{21}$

Within the Heusler compounds, several have been identified to be SGSs. ${ }^{8,22-24}$ Among them, an inverse Heusler compound, $\mathrm{Mn}_{2} \mathrm{CoAl}$, has attracted great attention since it is the only case where SGS behavior has been also confirmed experimentally. ${ }^{25} \mathrm{Mn}_{2} \mathrm{CoAl}$ crystallizes in a cubic structure known as the XA-structure similar to the $L 2_{1}$ structure of full Heusler compounds like $\mathrm{Co}_{2} \mathrm{MnAl}$ where only the sequence of the atoms in the unit cell changes (see Fig. 1 for a schematic representation of both structures) ${ }^{26,27} \mathrm{Mn}_{2} \mathrm{CoAl}$ was initially prepared experimentally by Liu and collaborators in 2008 (Ref. 28) but it was not until very recently that Ouardi et al. confirmed that $\mathrm{Mn}_{2} \mathrm{CoAl}$ is a SGS. ${ }^{25}$ The magnetic and transport properties of such films have been investigated in Ref. 29. First-principles calculations have confirmed the SGS character of $\mathrm{Mn}_{2} \mathrm{CoAl}^{22}$ and have established its connection to the magnetic properties. ${ }^{7}$ Moreover, recent studies on the effect of doping with $\mathrm{Co}, \mathrm{Cu}, \mathrm{V}$, and $\mathrm{Ti}^{30}$ as well as the anomalous Hall effect have appeared in the literature. ${ }^{31}$ The aim of the present study is to focus on the conditions for SGS behavior in $\mathrm{Mn}_{2} \mathrm{CoAl}$ compound. Employing the 


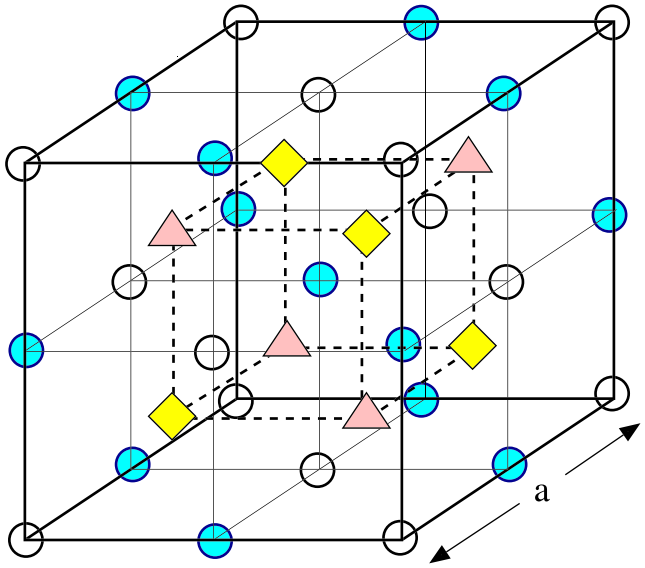

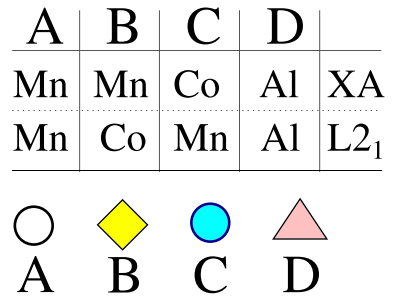

FIG. 1. Schematic representation of the cubic structure adopted by the $\mathrm{Mn}_{2} \mathrm{CoAl}$ and $\mathrm{Co}_{2} \mathrm{MnAl}$ compounds. The cube contains exactly four primitive unit cells. full-potential nonorthogonal local-orbital minimum-basis band structure scheme (FPLO), ${ }^{32}$ a state-of-the-art electronic structure method, we study the effect of (i) tetragonalization of the lattice, (ii) atomic swaps keeping the chemical stoichiometry, and (iii) surplus of Co atoms on the electronic and magnetic properties of $\mathrm{Mn}_{2} \mathrm{CoAl}$. In the case of Co surplus and atomic swaps, we employ the coherent-potential-approximation $(\mathrm{CPA})^{33}$ to simulate the disorder at different sites. As the exchange-correlation functional, we use the localspin-density approximation (LSDA) in all calculations. ${ }^{34}$ Note that LSDA is well-known to underestimate the equilibrium lattice constant with respect to generalized gradient approximation $(\mathrm{GGA})^{35}$ but for the same lattice constant it reproduces the GGA results concerning the density of states (DOS) giving small variations of the atomic spin moments keeping the same total spin magnetic moment in the unit cell. Thus, we use the experimental lattice of $5.8 \AA$ for $\mathrm{Mn}_{2} \mathrm{CoAl}$. The calculated total spin magnetic moments for the transition-metal atoms within LSDA deviate by less than $0.01 \mu_{\mathrm{B}}$ with respect to the GGA values keeping the same total spin magnetic moment in the unit cell of $2.0 \mu_{\mathrm{B}}$ and the SGS character. We expect these results to serve as a guideline to achieve experimentally SGS behavior in a variety of Heusler compound films proposed theoretically as SGS by first-principles calculations.

\section{TETRAGONALIZATION}

First, we have studied the effect of tetragonalization on the properties of the ordered $\mathrm{Mn}_{2} \mathrm{CoAl}$ in the absence of defects. We have varied the $c / a$ ratio taking into account both the cases of expansion and contraction of the $c$ lattice constant keeping the unit cell volume constant since this is the most usual case when films are grown on top of substrates with small lattice mismatch. Although we have

TABLE I. Atom resolved spin magnetic moments (in $\mu_{\mathrm{B}}$ ) for the perfect $\mathrm{Mn}_{2} \mathrm{CoAl}$ and for two cases of tetragonalization keeping the unit cell volume constant. Last column is the total spin magnetic moment per formula unit, which is integer in all cases.

\begin{tabular}{llllll}
\hline \hline $\mathrm{c} / \mathrm{a}$ & $\mathrm{Mn}^{\mathrm{A}}$ & $\mathrm{Mn}^{\mathrm{B}}$ & $\mathrm{Co}^{\mathrm{C}}$ & $\mathrm{Al}^{\mathrm{D}}$ & Total \\
\hline 0.95 & -1.847 & 2.940 & 0.953 & -0.046 & 2.000 \\
1.00 & -1.555 & 2.723 & 0.882 & -0.051 & 2.000 \\
1.05 & -1.291 & 2.520 & 0.825 & -0.054 & 2.000 \\
\hline \hline
\end{tabular}

considered several cases ( $c / a$ ratio changes by $\pm 0.5 \%, \pm 1 \%$, $\pm 2 \%$, and $\pm 5 \%$ ), we present in Table I results only for the two extreme cases since the atomic spin moments vary almost linearly with the $c / a$ ratio. When we compress the $c$ lattice constant by $5 \%$, the absolute values of the transition metal atoms are enhanced, while the opposite happens when we expand the $c$ lattice constant. Although the variation of the spin magnetic moments is important and can reach the $0.3 \mu_{\mathrm{B}}$ for the $\mathrm{Mn}$ atom at the A site, the total spin magnetic moment remains equal to $2 \mu_{\mathrm{B}}$ in all cases giving a hint that the SGS character is preserved.

In order to examine if the SGS behavior is also present in the distorted cases, one has to examine the band structure around the Fermi level since band crossing is not always easily distinguished in usual DOS calculations. In Fig. 2, we have plotted the spin-resolved band structure around the Fermi level for the perfect cubic lattice $(c / a=1$ in the middle panel) and the two cases also presented in Table I. In the middle panel for the perfect cubic lattice in the minority spin-down band structure, there is a direct energy gap at the $\Gamma$ point, which is created between the non-bonding $t_{1 u}$ and $e_{u}$ states as presented schematically in Figure 2 in Ref. 8 for the 26-valence electron Heusler compounds like $\mathrm{Mn}_{2} \mathrm{CoAl}$. In the majority spin-up band structure, there is an almost zero indirect energy gap created by the occupied band originating from the $e_{u}$ states, which has its maximum at the $\Gamma$ point, and the unoccupied $e_{g}$ band which has its minimum at the $\mathrm{X}$ point in the reciprocal space. (For a detailed discussion on the origin and character of the various bands, see Refs. 6-8.) One could argue that the material is not a true SGS since the Fermi level slightly crosses the top of the occupied majority-spin bands at $\Gamma$. But this is an artifact due to LSDA and the use of GGA establishes again the exact zero energy gap, ${ }^{22}$ and this imperceptible crossing is not affecting the validity of our conclusions. When we compress the $c$-axis (top panel) at the $\Gamma$ point, the top of the majority-spin band is shifted towards higher energy values crossing the Fermi level, while the bottom of the majority-spin unoccupied bands at the $\mathrm{X}$ point also crosses the Fermi level. These shifts are small and no real band crossing occurs. Thus, one can deduce that the SGS character is preserved. The energy gap in the minority spin band structure is slightly larger than the perfect cubic case. When we expand the $c$ lattice constant in the lower panel $(c / a=1.05)$, the bands move in the 

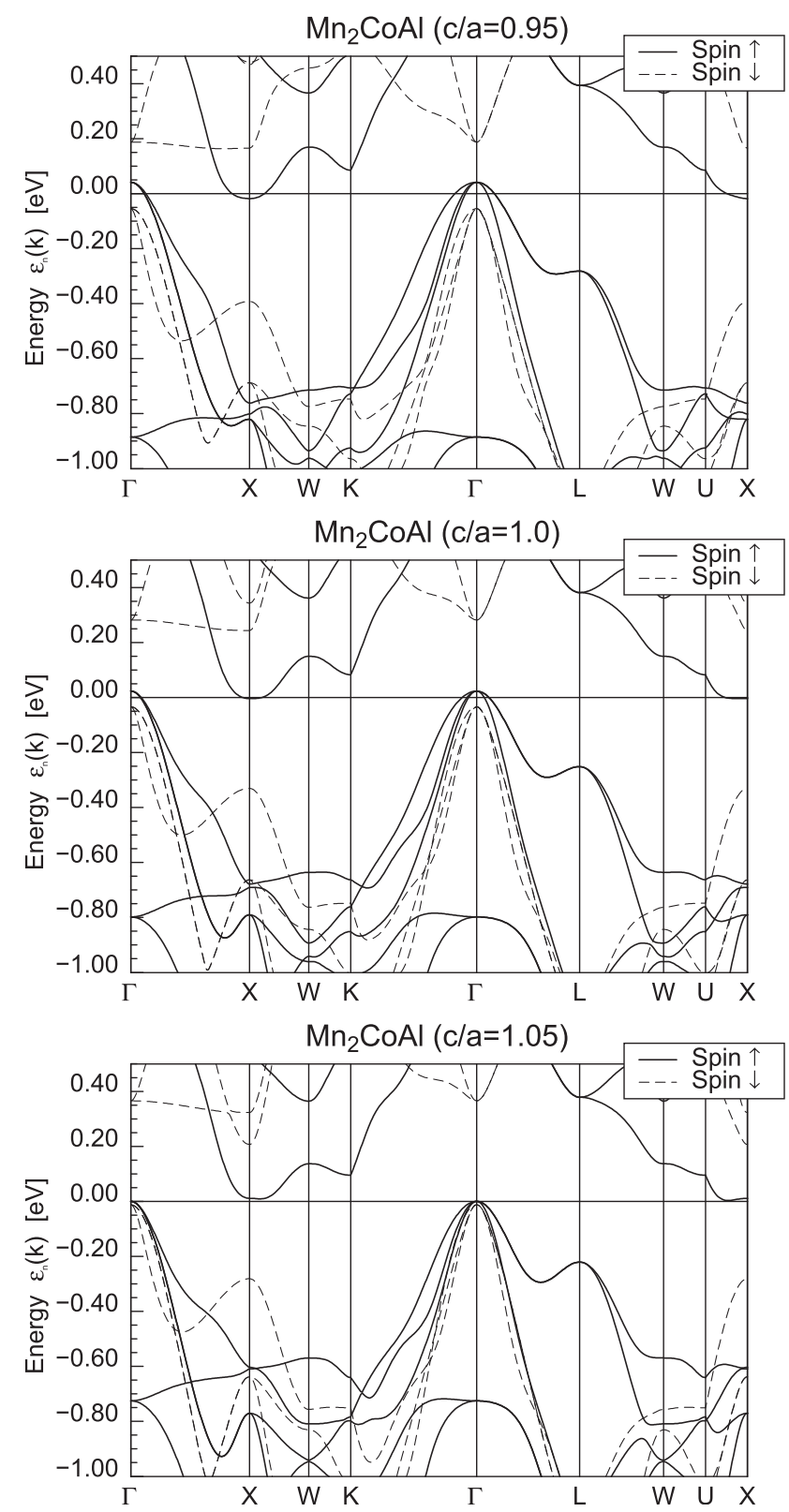

FIG. 2. Spin-resolved band structure of $\mathrm{Mn}_{2} \mathrm{CoAl}$ for the perfect cubic lattice $(c / a=1.0)$ in the middle and the case of contraction (expansion) in the upper (lower) panel. Note that $c / a$ changes keeping the unit cell volume constant. The zero energy has been chosen to represent the Fermi level. The solid (dashed) lines correspond to the majority spin-up (minority spin-down) states.

opposite direction with respect to the $c / a=0.95$ case but also in this case the SGS character is preserved. Thus, even large variation of the $c / a$ ratio does not destroy the SGS character of the parent cubic compound.

\section{ATOMIC SWAPS}

Next, we study the effect of defects keeping the stoichiometry since these are the most likely to occur. There are two cases of such atomic swaps (i) the ones when atoms move to sites with the same local environment (same nearest neighbors) and (ii) when the impurity atoms have a different local environment with respect to their initial site. In the cubic structure adopted by the Heusler compounds presented

TABLE II. Atom resolved spin magnetic moments (in $\mu_{\mathrm{B}}$ ) for the impurity cases, where the local symmetry is conserved. Last column is the total spin magnetic moment per formula unit.

\begin{tabular}{|c|c|c|c|c|c|c|c|}
\hline \multicolumn{8}{|c|}{$\left(\mathrm{Mn}_{1-x} \mathrm{Co}_{x}\right) \mathrm{Mn}\left(\mathrm{Co}_{1-x} \mathrm{Mn}_{x}\right) \mathrm{Al}$} \\
\hline $\mathrm{x}$ & $\mathrm{Mn}^{\mathrm{A}}$ & $\mathrm{Co}_{\mathrm{imp}}^{\mathrm{A}}$ & $\mathrm{Mn}^{\mathrm{B}}$ & $\mathrm{Co}^{\mathrm{C}}$ & $\mathrm{Mn}_{\mathrm{imp}}^{\mathrm{C}}$ & $\mathrm{Al}^{\mathrm{D}}$ & Total \\
\hline 0.005 & -1.540 & 0.862 & 2.707 & 0.888 & -1.789 & -0.053 & 2.000 \\
\hline 0.010 & -1.539 & 0.834 & 2.709 & 0.886 & -1.804 & -0.052 & 2.000 \\
\hline 0.020 & -1.536 & 0.824 & 2.712 & 0.882 & -1.824 & -0.051 & 2.000 \\
\hline 0.050 & -1.530 & 0.828 & 2.722 & 0.874 & -1.850 & -0.048 & 2.000 \\
\hline \multicolumn{8}{|c|}{$\operatorname{Mn}\left(\mathrm{Mn}_{1-x} \mathrm{Al}_{x}\right) \operatorname{Co}\left(\mathrm{Al}_{1-x} \mathrm{Mn}_{x}\right)$} \\
\hline $\mathrm{x}$ & $\mathrm{Mn}^{\mathrm{A}}$ & $\mathrm{Mn}^{\mathrm{B}}$ & $\mathrm{Al}_{\mathrm{imp}}^{\mathrm{B}}$ & $\mathrm{Co}^{\mathrm{C}}$ & $\mathrm{Al}^{\mathrm{D}}$ & $\mathrm{Mn}_{\mathrm{imp}}^{\mathrm{D}}$ & Total \\
\hline 0.005 & -1.539 & 2.708 & 0.008 & 0.884 & -0.053 & 2.913 & 2.000 \\
\hline 0.010 & -1.537 & 2.709 & 0.006 & 0.879 & -0.053 & 2.891 & 2.000 \\
\hline 0.020 & -1.534 & 2.712 & 0.003 & 0.870 & -0.052 & 2.884 & 2.000 \\
\hline 0.050 & -1.523 & 2.719 & -0.000 & 0.848 & -0.051 & 2.886 & 2.000 \\
\hline
\end{tabular}

in Fig. 1, the atoms sitting at the A and $\mathrm{C}$ sites (or at the B and $\mathrm{D}$ sites) have the same local environment since they have the same nearest neighbors. Thus, to the first category of studied defects either Mn at the A site and Co atoms at the $\mathrm{C}$ site migrate one to another or Mn atoms at the B sites and $\mathrm{Al}$ atoms at the $\mathrm{D}$ sites exchange sites. We present our calculated results for these cases in Table II. In both cases under study, the atom-resolved spin magnet moments change slightly with the concentration of the defects and the impurity atoms have atomic spin magnetic moments comparable to the ones at their initial site. We expect that such defects will not influence the character of the bands in the two spin directions and the Slater-Pauling rule in Ref. 7 will be still valid. Our calculations confirm this assumption and overall the total spin magnetic moment is kept equal to $2 \mu_{\mathrm{B}}$. This integer value suggests that the alloys under study are halfmetals but it does not guarantee that SGS is present. A close look at the DOS around the Fermi level presented in Fig. 3 for the cases with the largest percentage of atomic swaps reveals that the SGS behavior is not conserved. In the majority spin band structure around the Fermi level, there is a region of low DOS intensity instead of a zero-width energygap. Thus, these compounds are half-metals but not SGS.

Most interesting are the cases where atomic swaps occur between sites with different local symmetry. In this case, bands with different character are mixed in the disordered compound and the Slater-Pauling rule connecting the SGS or HM behavior to the total spin magnetic moment in the formula unit is no more valid. ${ }^{7}$ In Table III, we have gathered our results for the cases of $\mathrm{Mn}(\mathrm{A})-\mathrm{Al}(\mathrm{D}), \mathrm{Co}(\mathrm{C})-\mathrm{Al}(\mathrm{D})$, and $\mathrm{Mn}(\mathrm{B})-\mathrm{Co}(\mathrm{C})$ swaps, where in parenthesis are the sites involved in the swaps. The spin magnetic moments of the impurity atoms deviate strongly from the ones at the initial site. For example, in the case of $\left(\mathrm{Mn}_{1-x} \mathrm{Al}_{x}\right) \mathrm{MnCo}\left(\mathrm{Al}_{1-x} \mathrm{Mn}_{x}\right)$ alloys the Mn impurity atoms at the D site have a spin magnetic moment about $2.9 \mu_{\mathrm{B}}$. The latter is close to the spin moment of Mn atoms at the B site (about $2.7 \mu_{\mathrm{B}}$ ) since B and $\mathrm{D}$ sites have the same local symmetry and deviates strongly from the Mn spin moment at the initial A site where the Mn 


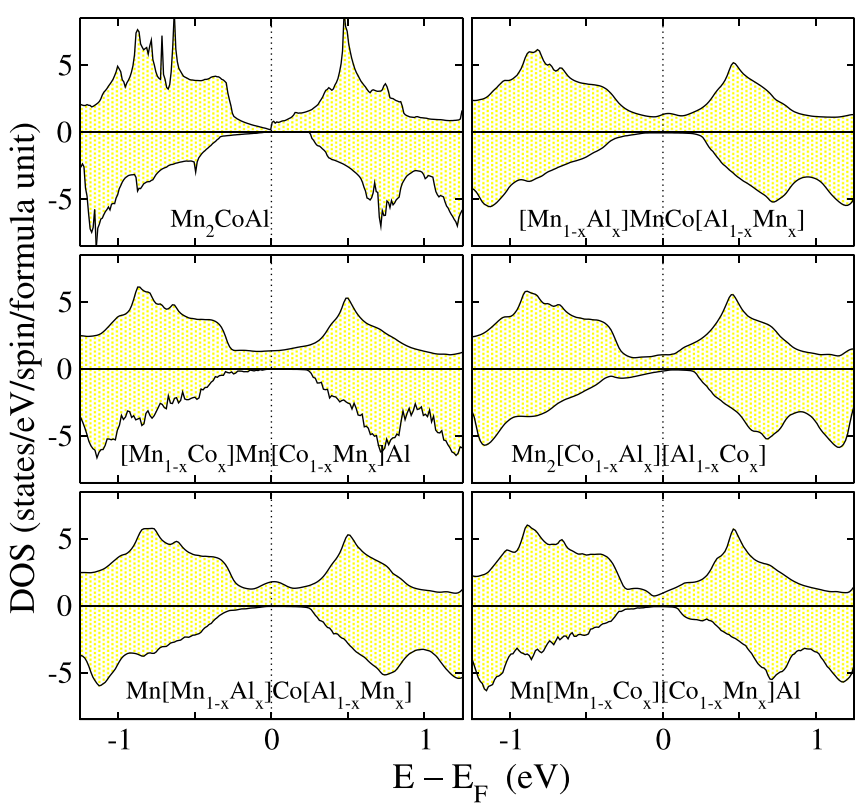

FIG. 3. Total DOS (per formula unit) for the perfect $\mathrm{Mn}_{2} \mathrm{CoAl}$ compounds and the impurity cases presented in Tables II and III (cases where $\mathrm{x}=0.05$ ). Positive (negative) DOS values correspond to the spin-up (spin-down) electrons. The zero energy corresponds to the Fermi level.

spin moments are negative and close to $-1.5 \mu_{\mathrm{B}}$. In all three cases, the total spin magnetic moment per formula unit deviates from the ideal $2 \mu_{\mathrm{B}}$ values since, as we mentioned above, the Slater-Pauling rule is no more valid. A close look at the variation of the total spin magnetic moment in Table III reveals that for all three cases this variation is linear with the percentage of the swaps $x$ and even more interestingly

TABLE III. Atom resolved spin magnetic moments (in $\mu_{\mathrm{B}}$ ) for the impurity cases, where the local symmetry is not-conserved and therefore the SlaterPauling rule is no more valid. ${ }^{7}$ Last column is the total spin magnetic moment per formula unit.

\begin{tabular}{lccccccc}
\hline \hline \multicolumn{7}{c}{$\left(\mathrm{Mn}_{1-x} \mathrm{Al}_{x}\right) \mathrm{MnCo}\left(\mathrm{Al}_{1-x} \mathrm{Mn}_{x}\right)$} \\
\hline $\mathrm{x}$ & $\mathrm{Mn}^{\mathrm{A}}$ & $\mathrm{Al}_{\mathrm{imp}}^{\mathrm{A}}$ & $\mathrm{Mn}^{\mathrm{B}}$ & $\mathrm{Co}^{\mathrm{C}}$ & $\mathrm{Al}^{\mathrm{D}}$ & $\mathrm{Mn}_{\text {imp }}^{\mathrm{D}}$ & Total \\
\hline 0.005 & -1.538 & -0.082 & 2.707 & 0.892 & -0.054 & 2.946 & 2.030 \\
0.010 & -1.534 & -0.083 & 2.708 & 0.895 & -0.054 & 2.922 & 2.060 \\
0.020 & -1.526 & -0.084 & 2.710 & 0.902 & -0.054 & 2.918 & 2.120 \\
0.050 & -1.503 & -0.086 & 2.716 & 0.922 & -0.056 & 2.940 & 2.300
\end{tabular}

$\operatorname{MnMn}\left(\mathrm{Co}_{1-x} \mathrm{Al}_{x}\right)\left(\mathrm{Al}_{1-x} \mathrm{Co}_{x}\right)$

\begin{tabular}{lccccccc}
\hline $\mathrm{x}$ & $\mathrm{Mn}^{\mathrm{A}}$ & $\mathrm{Mn}^{\mathrm{B}}$ & $\mathrm{Co}^{\mathrm{C}}$ & $\mathrm{Al}_{\text {imp }}^{\mathrm{C}}$ & $\mathrm{Al}^{\mathrm{D}}$ & $\mathrm{Co}_{\text {imp }}^{\mathrm{D}}$ & Total \\
\hline 0.005 & -1.546 & 2.702 & 0.891 & -0.012 & -0.053 & -1.869 & 1.980 \\
0.010 & -1.551 & 2.700 & 0.891 & -0.011 & -0.053 & -1.858 & 1.960 \\
0.020 & -1.560 & 2.695 & 0.892 & -0.009 & -0052 & -1.840 & 1.921 \\
0.050 & -1.590 & 2.678 & 0.899 & -0.005 & -0.050 & -1.790 & 1.805
\end{tabular}

$\mathrm{Mn}\left(\mathrm{Mn}_{1-x} \mathrm{Co}_{x}\right)\left(\mathrm{Co}_{1-x} \mathrm{Mn}_{x}\right) \mathrm{Al}$

\begin{tabular}{lccccccc}
\hline $\mathrm{x}$ & $\mathrm{Mn}^{\mathrm{A}}$ & $\mathrm{Mn}^{\mathrm{B}}$ & $\mathrm{Co}_{\text {imp }}^{\mathrm{B}}$ & $\mathrm{Co}^{\mathrm{C}}$ & $\mathrm{Mn}_{\text {imp }}^{\mathrm{C}}$ & $\mathrm{Al}^{\mathrm{D}}$ & Total \\
\hline 0.005 & -1.538 & 2.706 & 0.214 & 0.891 & -1.774 & -0.053 & 1.980 \\
0.010 & -1.535 & 2.706 & 0.213 & 0.892 & -1.775 & -0.052 & 1.960 \\
0.020 & -1.528 & 2.709 & 0.212 & 0.893 & -1.776 & -0.050 & 1.920 \\
0.050 & -1.508 & 2.714 & 0.211 & 0.895 & -1.737 & -0.045 & 1.800 \\
\hline \hline
\end{tabular}

the DOS presented in Fig. 3 show a half-metallic behavior with an energy gap around the Fermi level in the minority spin gap. Half-metallicity, when present, is a particular stable state since exchange constants for half-metals strongly support the appearance of magnetism. ${ }^{36}$ Thus, these atomic swaps change the total spin magnetic moment but the large exchange splitting of the occupied and unoccupied $d$-states of different spin-character at each transition metal site keeps the half metallic character of the disordered compound. The linear variation of the total spin magnetic moment with the concentration $x$ of the swaps is due to the fact that as shown in Table III the atomic spin moments are almost independent from $x$ and thus the total spin moment, which is the weighted sum of all atomic spin moments changes linearly with $x$.

\section{Co-SURPLUS}

In the final step, we have concentrated on the case of Cosurplus. $\mathrm{Co}_{2} \mathrm{MnAl}$ is a well-studied half-metallic ferromagnetic Heusler compound with a lattice constant of $a=5.78 \AA^{37}$ As shown in Fig. 1, we can move from $\mathrm{Mn}_{2} \mathrm{CoAl}$ to $\mathrm{Co}_{2} \mathrm{MnAl}$ in a continuous way just by substituting Co for the Mn atoms at the A site. In Fig. 4, we show the variation of the atomic spin moments at the various sites (scaled to one atom) as well as the total one per formula unit as a function of the concentration $x$ in the $\left(\mathrm{Mn}_{1-x} \mathrm{Co}_{x}\right) \mathrm{MnCoAl}$ compound. The Co impurity atoms at the A site have spin moments comparable to the Co atoms at the $\mathrm{C}$ site since they have the same local environment. Also, the Mn atom at the B site presents a constant spin magnetic moment almost irrespectively of the $x$ values. The absolute value of the spin magnetic moments of the Mn atoms at the A sites increases but since their percentage in the compounds decreases as $x$ increases, their weight in the total spin magnetic moment decreases rapidly. The total spin magnetic changes linearly from the $2 \mu_{\mathrm{B}}$ of the perfect $\mathrm{Mn}_{2} \mathrm{CoAl}(x=0)$ to the $4 \mu_{\mathrm{B}}$ of the perfect $\mathrm{Co}_{2} \mathrm{MnAl}(x=1)$. A close look at the DOS, not presented here, reveals that all intermediate compounds are half-metallic and the energy gap in the minority-spin band structure persists for all $x$-values. But the SGS behavior is a

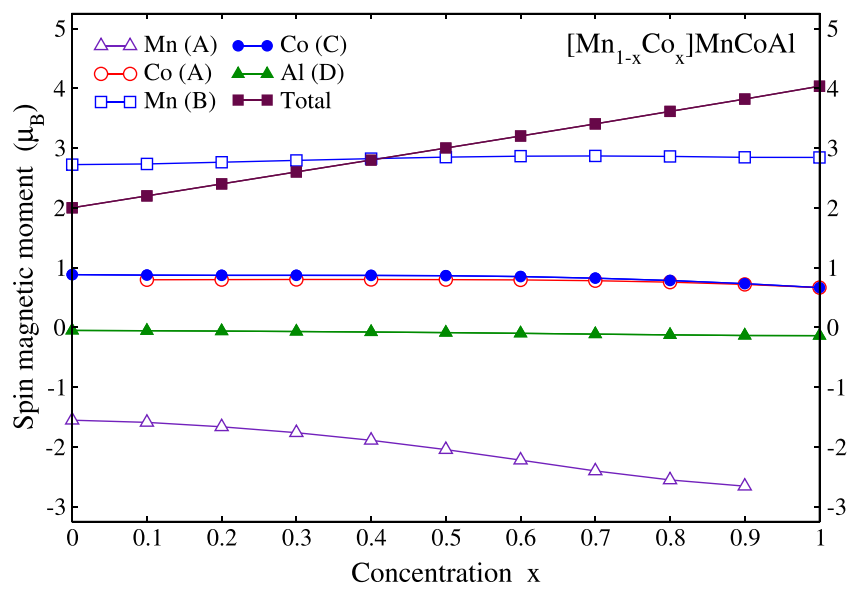

FIG. 4. Spin magnetic moment as a function of the concentration as we dope the A site in $\mathrm{Mn}_{2} \mathrm{CoAl}$ with Co. Note that for all the intermediate concentrations, the total spin magnetic moment in the formula unit follows the Slater-Pauling rule. 
characteristic only of $\mathrm{Mn}_{2} \mathrm{CoAl}$ since even a small percentage of Co atoms at the A site leads to the disappearance of the zero-energy gap in the majority spin band structure.

\section{CONCLUSIONS}

Employing ab initio electronic structure calculation, we have investigated the conditions for SGS behavior in $\mathrm{Mn}_{2} \mathrm{CoAl}$ compound. First, tetragonalization of the lattice, which can occur during growth on various substrates, keeps the SGS character of the perfect cubic compound. Second, atomic swaps even between sites with different local symmetry lead to half-metallicity since the zero-energy gap in the majority-spin band-structure vanishes, and finally, the occurrence Co-surplus during growth leads also to half-metallicity as one goes from $\mathrm{Mn}_{2} \mathrm{CoAl}$ to $\mathrm{Co}_{2} \mathrm{MnAl}$. Thus, to get SGS behavior during the growth of $\mathrm{Mn}_{2} \mathrm{CoAl}$, one should minimize the occurrence of defects, while small deformations of the lattice, due to the lattice mismatch with the substrate, do not play a crucial role. We expect these results to trigger further experimental interest in growing a variety of Heusler compounds predicted to be SGS by first-principles calculations.

\section{ACKNOWLEDGMENTS}

K.Ö. acknowledges the support from YTU through Grant No. 2012-01-01-KAP08.

${ }^{1}$ C. Felser, G. H. Fecher, and B. Balke, Angew. Chem., Int. Ed. 46, 668 (2007).

${ }^{2}$ R. A. de Groot, F. M. Mueller, P. G. van Engen, and K. H. J. Buschow, Phys. Rev. Lett. 50, 2024 (1983).

${ }^{3}$ M. I. Katsnelson, V. Yu. Irkhin, L. Chioncel, A. I. Lichtenstein, and R. A. de Groot, Rev. Mod. Phys. 80, 315 (2008).

${ }^{4}$ I. Žutić, J. Fabian, and S. Das Sarma, Rev. Mod. Phys. 76, 323 (2004).

${ }^{5}$ I. Galanakis, P. H. Dederichs, and N. Papanikolaou, Phys. Rev. B 66, 134428 (2002).

${ }^{6}$ I. Galanakis, P. H. Dederichs, and N. Papanikolaou, Phys. Rev. B 66, 174429 (2002).

${ }^{7}$ S. Skaftouros, K. Özdoğan, E. Şaşıoğlu, and I. Galanakis, Phys. Rev. B 87, 024420 (2013).

${ }^{8}$ K. Özdoğan, E. Şaşığlu, and I. Galanakis, J. Appl. Phys. 113, 193903 (2013).
${ }^{9}$ I. Galanakis, K. Özdoğan, and E. Şaşığlu, Appl. Phys. Lett. 103, 142404 (2013); I. Galanakis, K. Özdoğan, and E. Şaşıoğlu, J. Phys.: Condens. Matter 26, 086003 (2014).

${ }^{10}$ I. Galanakis and E. Şaşioğlu, Appl. Phys. Lett. 99, 052509 (2011).

${ }^{11}$ I. M. Tsidilkovski, Electron Spectrum of Gapless Semiconductors, Springer Series in Solid-State Sciences Vol. 116, edited by K. von Klitzing (Springer, New York, 1996).

${ }^{12}$ J. A. Kurzman, M.-S. Miao, and R. Seshadri, J. Phys.: Condens. Matter 23, 465501 (2011).

${ }^{13}$ S. W. Chen, S. C. Huang, G. Y. Guo, J. M. Lee, S. Chiang, W. C. Chen, Y. C. Liang, K. T. Lu, and J. M. Chen, Appl. Phys. Lett. 99, 012103 (2011).

${ }^{14}$ K. S. Novoselov, A. K. Geim, S. V. Morozov, D. Jiang, Y. Zhang, S. V. Dubonos, I. V. Grigorieva, and A. A. Firsov, Science 306, 666 (2004).

${ }^{15}$ X. L. Wang, Phys. Rev. Lett. 100, 156404 (2008); X. Wang, G. Peleckis, C. Zhang, H. Kimura, and S. Dou, Adv. Mater. 21, 2196 (2009).

${ }^{16}$ D. H. Kim, J. Hwang, E. Lee, K. J. Lee, S. M. Choo, M. H. Jung, J. Baik, H. J. Shin, B. Kim, K. Kim, B. I. Min, and J.-S. Kang, Appl. Phys. Lett. 104, 022411 (2014).

${ }^{17}$ Y. Pan and Z. Yang, Chem. Phys. Lett. 518, 104 (2011).

${ }^{18}$ Z. F. Wang, S. Jin, and F. Liu, Phys. Rev. Lett. 111, 096803 (2013).

${ }^{19}$ S.-D. Guo and B.-G. Liu, J. Phys.: Condens. Matter 24, 045502 (2012).

${ }^{20}$ Y. Pan and Z. Yang, Phys. Rev. B 82, 195308 (2010).

${ }^{21}$ J. He, P. Zhou, N. Jiao, L. Z. Sun, X. Chen, and W. Lu, e-print arXiv: 1308.0253 .

${ }^{22}$ S. Skaftouros, K. Özdoğan, E. Şaşığlu, and I. Galanakis, Appl. Phys. Lett. 102, 022402 (2013).

${ }^{23}$ G. Z. Xu, E. K. Liu, Y. Du, G. J. Li, G. D. Liu, W. H. Wang, and G. H. Wu, Europhys. Lett. 102, 17007 (2013).

${ }^{24}$ G. Y. Gao and K.-L. Yao, Appl. Phys. Lett. 103, 232409 (2013).

${ }^{25}$ S. Ouardi, G. H. Fecher, C. Felser, and J. Kübler, Phys. Rev. Lett. 110, 100401 (2013).

${ }^{26}$ K. Özdoğan and I. Galanakis, J. Magn. Magn. Mater. 321, L34 (2009).

${ }^{27}$ K. Özdoğan and I. Galanakis, J. Appl. Phys. 110, 076101 (2011).

${ }^{28}$ G. D. Liu, X. F. Dai, H. Y. Liu, J. L. Chen, Y. X. Li, G. Xiao, and G. H. Wu, Phys. Rev. B 77, 014424 (2008).

${ }^{29}$ M. E. Jamer, B. A. Assaf, T. Devakul, and D. Heiman, Appl. Phys. Lett. 103, 142403 (2013).

${ }^{30}$ Y. J. Zhang, G. J. Li, E. K. Liu, J. L. Chen, W. H. Wang, and G. H. Wu, J. Appl. Phys. 113, 123901 (2013).

${ }^{31}$ J. Kudrnovský, V. Drchal, and I. Turek, Phys. Rev. B 88, 014422 (2013).

${ }^{32}$ K. Koepernik and H. Eschrig, Phys. Rev. B 59, 1743 (1999).

${ }^{33}$ K. Koepernik, B. Velicky, R. Hayn, and H. Eschrig, Phys. Rev. B 55, 5717 (1997); 58, 6944 (1998).

${ }^{34}$ J. P. Perdew and Y. Wang, Phys. Rev. B 45, 13244 (1992).

${ }^{35}$ J. P. Perdew, K. Burke, and M. Ernzerhof, Phys. Rev. Lett. 77, 3865 (1996).

${ }^{36}$ I. Galanakis and E. Şaşıŏlu, J. Appl. Phys. 109, 113912 (2011).

${ }^{37}$ S. Trudel, O. Gaier, J. Hamrle, and B. Hillerbrands, J. Phys. D: Appl. Phys. 43, 193001 (2010). 